\title{
Intraoperative Neurological Injury, CTCAE
}

National Cancer Institute

\section{Source}

National Cancer Institute. Intraoperative Neurological Injury, CTCAE. NCI Thesaurus.

Code C143607.

A finding of damage to the nervous system during a surgical procedure. 